\title{
Optical Resolution of 1,2-Diamino Compounds Using Advanced Marfey's Method
}

\author{
Yuriko Nozawa, ${ }^{1}$ Ken-ichi Harada, ${ }^{1 *}$ Akemi Ryoda, ${ }^{2}$ Takuya Kumamoto, ${ }^{2}$ \\ Waka NAKANISHI, ${ }^{2}$ and Tsutomu IsHIKAWA ${ }^{2}$ \\ ${ }^{1}$ Graduate School of Environmental and Human Science, and Faculty of Pharmacy, Meijo University, \\ Nagoya, JAPAN \\ ${ }^{2}$ Graduate School of Pharmaceutical Sciences, Chiba University, Chiba, JAPAN
}

\begin{abstract}
Advanced Marfey's method was successfully applied to the optical resolution of 1,2-diaminoethane compounds. The elution behavior of 1,2-diphenylethylene-1,2-diamine and 1,2-bis(2-methylphenyl)ethylene-1,2diamine derivatized with L- and D-FDLA was carefully investigated and the following regularity was found: 1$)$ The $(S, S)$-isomers are eluted prior to the $(R, R)$-isomer; 2$)$ The $(R, R)$ - and meso-isomers are closely eluted; 3$)$ There is no difference between the retention time of both the L- and D-derivatives of the meso-isomer; 4) This regularity is fundamentally consistent with that of diamino acids such as diaminopimelic acid and diaminosuccinic acid. Finally, it was confirmed that this method along with the DL-FDLA method could be applicable for the determination of the absolute configuration of 1,2-diaminoethane compounds because the method can definitely determine it by the elution order.
\end{abstract}

(Received January 5, 2009; Accepted January 26, 2009)

\section{Introduction}

Advanced Marfey's method has been developed to non-empirically determine the absolute configurations of the constituent amino acids in a peptide using liquid chromatography/mass spectrometry (LC/MS). ${ }^{1), 2)}$ In this method, the derivatized D- and L-amino acids with 1-fluoro-2,4-dinitrophenyl-5-L-leucinamide (L-FDLA) are analyzed by LC/MS, and the absolute configuration of the D- and L-amino acids can be determined from the elution behavior of the 2,4-dinitrophenyl-5-L-leucinamide (L-DLA) derivatives under reversed phase conditions. Furthermore, advanced Marfey's method using 1-fluoro-2,4-dinitrophenyl-5-DL-leucinamide (DL-FDLA) derivatization can provide the corresponding enantiomer from either the L- or D-amino acid in the chromatogram without the conventional chemical racemization of an amino acid.

Ordinarily, chromatographic methods such as gas chromatography (GC) and high-performance liquid chromatography (HPLC) have been widely used for the determination of the absolute configuration of amino acids. In most cases, it is determined by comparison with the retention time of a standard amino acid under diastereomeric circumstance after appropriate derivatization. On the other hand, advanced Marfey's method does not necessarily require a standard amino acid, since the resultant derivatives can be detected using

* Corresponding to: Ken-ichi HARADA, Graduate School of Environmental and Human Science, and Faculty of Pharmacy, Meijo University, Tempaku, Nagoya 468-8503, JAPAN, e-mail: kiharada@ccmfs.meijo-u.ac.jp

This paper is dedicated to the late Dr. Hiroshi Miyazaki in honor of his many contributions to our field.
LC/MS. The operation can be performed with very small amount of an amino acid without any interference by other amino acids.

In a previous study, we investigated the elution behavior of diaminopimelic acid $\left(\mathrm{A}_{2} \mathrm{pm}\right)$ by advanced Marfey's method using actinomycetal standard strains that are known to contain the LL- $\mathrm{A}_{2} \mathrm{pm}$ or meso- $\mathrm{A}_{2} \mathrm{pm}$ and commercially available $\mathrm{A}_{2} \mathrm{pm}$ composed of three isomers, LL-, DD-, and meso- $\mathrm{A}_{2} \mathrm{pm}^{3)}$ As a result, we determined the elution order of the L-DLA derivatives of the $\mathrm{A}_{2} \mathrm{pm}$ isomers as follows: meso-, LL-, and DD- $\mathrm{A}_{2} \mathrm{pm}$. However, excluding this case, no determination of the absolute configuration of the amino acid with a "meso" configuration has been performed by this method.

Advanced Marfey's method in combination with chemical racemization and DL-FDLA methods ${ }^{4)}$ was further used to determine the absolute configuration of several diamino acids including $\mathrm{A}_{2} \mathrm{pm}$ with a "meso" configuration. ${ }^{5)}$ Based on the elution order, the absolute configuration was definitely determined. In our previous paper, we also investigated the elution behavior of the derivatized diamino acids with $\mathrm{L}$ - and D-FDLA, and the following regularity for the L-DLA derivatives was found: 1) The LL- and meso-isomers are eluted prior to the DD-isomer except for one case; 2) The LL- and meso-isomers are closely eluted and occasionally the elution is reversed; 3) There is no difference between the retention time for both the $\mathrm{L}^{-}$and $\mathrm{D}$ derivatives of the meso-isomer. This regularity was explained using the separation mechanism for advanced Marfey's method. ${ }^{2)}$ Throughout this study, it was confirmed that advanced Marfey's method can be used for determination of the absolute configuration of the diamino acid in addition to the usual amino acid.

The trans-1,2-diaminoethane compounds have been 


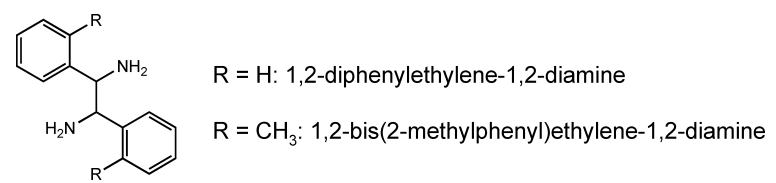

Fig. 1. Structures of 1,2-diphenylethylene-1,2-diamine and 1,2-bis(2-methylphenyl)ethylene-1,2-diamine.

recognized as the very useful frameworks of chiral catalysts, scaffolds, and ligands for asymmetric synthesis and molecular recognition. ${ }^{6)-8)}$ Ishikawa et al. ${ }^{91-18)}$ designed several types of guanidine compounds with chiral center(s) based on 1,2-diphenylethylene-1,2diamine and found that 2-(1-benzyl-2-hydroxyethyl)im ino-1,3-dimethy -4,5-diphenylimidazolidine (cyclic guanidine) acts as an efficient chiral catalyst for the asymmetric Michael reaction of tert-butyl diphenyliminoacetate and active vinyl compounds such as methyl vinyl ketone and acrylate. ${ }^{14)}$ For further optimization of this guanidine catalyst, structural modification of the phenyl pendant on the imidazolidine ring was carried out and produced trans-1,2-bis(2methylphenyl)ethylene-1,2-diamine as a new chiral framework. ${ }^{19)}$ The optically pure derivative was prepared by the optical resolution of the racemic compound and the absolute configuration was fortunately determined by an X-ray crystallographic analysis. However, a general and simple evaluation method of the optical purity for this type of diamine compound has been strongly requested.

We have shown that advanced Marfey's method can be successfully applied to the determination of the absolute configuration of primary amino compounds and its utility was extended from amino acids to primary amino compounds. ${ }^{20)}$ In this study, the method was applied to the 1,2-diaminoethane compounds (Fig. 1) and the potential was carefully investigated for the purpose described above.

\section{Experimental}

\subsection{Materials}

The derivatizing reagents, L-FDLA, 1-fluoro-2,4-dinitrophenyl-5-D-leucinamide (D-FDLA), and 1,2-diphenylethylene-1,2-diamine were purchased from Tokyo Chemical Industry Co., Ltd. (Tokyo, Japan). 1,2-Bis(2methylphenyl)ethylene-1,2-diamine were prepared from 2-methylbenzoyl chloride according to the method reported by Ishikawa et al. ${ }^{19)}$

\subsection{Sample preparation}

The derivatization of the diamino compounds with Lor D-FDLA was basically performed according to the method described in a previous paper ${ }^{1)}$ : Ten $\mu \mathrm{L}$ of $1 \mathrm{M}$ sodium bicarbonate and then $50 \mu \mathrm{L}$ of $1 \%$ FDLA in acetone were added to $25 \mu \mathrm{L}$ of a $50 \mathrm{mM}$ aqueous solution of diamino compounds. The solution was vortexed and incubated at $37^{\circ} \mathrm{C}$ for $1 \mathrm{~h}$ or $24 \mathrm{~h}$. The reaction was quenched by the addition of $10 \mu \mathrm{L}$ of $1 \mathrm{M}$ $\mathrm{HCl}$. Ten $\mu \mathrm{L}$ of the reaction mixture was diluted with $90 \mu \mathrm{L}$ of acetonitrile, and $10 \mu \mathrm{L}$ of this diluted solution was then analyzed by electrospray ionization (ESI) LC/ MS.

\subsection{ESI LC/MS conditions}

The separation of the L- and D-DLA derivatives of the diamino compounds was performed using a Model 1,100 liquid chromatograph system (Agilent, California, USA). The separations were carried out using a YMC-Pack Pro C18 $(100 \times 4.6 \mathrm{~mm}$ i.d., $3 \mu \mathrm{m}$, YMC $)$ column at $40^{\circ} \mathrm{C}$. Acetonitrile-water containing $5 \mathrm{mM}$ of ammonium formate and $5 \mathrm{mM}$ of formic acid was used as the mobile phase under a linear gradient elution mode (acetonitrile, 15-80\%, $20 \mathrm{~min}$ ) at the flow rate of $1.0 \mathrm{~mL} / \mathrm{min}$ with ultraviolet (UV) detection at $340 \mathrm{~nm}$ and $210-600 \mathrm{~nm}$ by photodiode array detection. The Agilent 1,100 series single quadruple mass spectrometer (G1946D) was used for the ESI mass analysis. The mass conditions were follows: For mass spectrometry (MS) detection, the ESI negative ionization mode was selected. The MS capillary voltage was set to $2,000 \mathrm{~V}$ and the MS fragmentor voltage was set to $100 \mathrm{~V}$ throughout this study. The nebulizer gas, the drying gas flow rate, and the temperature were set to $45 \mathrm{psi}, 13 \mathrm{~L} / \mathrm{min}$, and $350^{\circ} \mathrm{C}$, respectively. The mass chromatography was used for the detection of the stereoisomers of 1,2-diphenylethylenediamine $(\mathrm{m} / z 799$ $\left.[\mathrm{M}-\mathrm{H}]^{-}\right)$, and 1,2-di(2-methylphenyl)-ethylenediamine $\left(m / z 827[\mathrm{M}-\mathrm{H}]^{-}\right)$.

\section{Results and Discussion}

\subsection{Elution behavior of 1,2-diphenylethylene-1,2- diamine derivatized with FDLA}

The available $(S, S)-, \quad(R, R)-, \quad(d l)_{-}$, and meso-1,2diphenylethylene-1,2-diamines were derivatized with L-FDLA for $1 \mathrm{~h}$. The mass current chromatograms of the $[\mathrm{M}-\mathrm{H}]^{-}$at $m / z 799$ of the derivatives are shown in Fig. 2. The desired peaks are clearly observed and separated, and the elution order of the L-DLA derivatives was as follows: $(S, S) \rightarrow$ meso $\rightarrow(R, R)$. The DL-FDLA method is quite effective for obtaining the corresponding enantiomers of an amino acid and was applied to these isomers. Figure 3 shows the mass ion current chromatograms of the $[\mathrm{M}-\mathrm{H}]^{-}$at $m / z 799$ of the amines derivatized with D-FDLA. While the retention time for the $(S, S)$-derivative with L-FDLA was consistent with that of the $(R, R)$-derivative with D-FDLA, that for both the $\mathrm{L}$ - and $\mathrm{D}$-derivatives of the mesoisomer was not changed (Figs. 2 and 3).

We previously investigated the elution behavior of the diaminosuccinic acid derivatized with FDLA and the following results were obtained: the combination of the chemical racemization and DL-FDLA method could be successfully applied, the resulting elution order of the $\mathrm{L}-\mathrm{DLA}$ derivatives was $(\mathrm{L}, \mathrm{L}) \rightarrow$ meso $\rightarrow(\mathrm{D}, \mathrm{D})$ and there is no difference between the retention time of both the L- and D-derivatives of the meso-isomer. ${ }^{5}$ These results suggest that advance Marfey's method could be applied to the 1,2-diamino compounds and used to determine the absolute configuration of these types of compounds.

\subsection{Elution behavior of 1,2-bis(2-methylphenyl)} ethylene-1,2-diamine derivatized with FDLA

As described in the introduction, 1,2-bis(2-methylphenyl)ethylene-1,2-diamine is very important as the frameworks of chiral catalysts, scaffolds, and ligands for asymmetric synthesis and molecular recognition. Although the enantioselective syntheses have been achieved, ${ }^{21,22)}$ their optical rotations were not reported, 

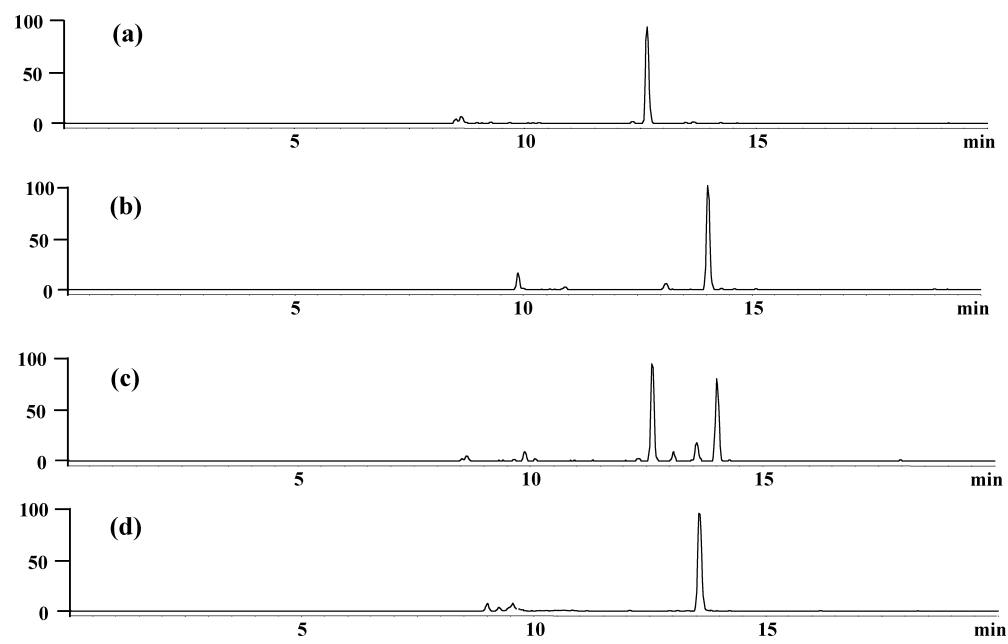

Fig. 2. Mass chromatograms of the $[\mathrm{M}-\mathrm{H}]^{-}$at $\mathrm{m} / z 799$ for (a) $(\mathrm{S}, \mathrm{S})$-diphenylethylenediamine derivatized with L-FDLA; (b) $(R, R)$-diphenylethylenediamine derivatized with L-FDLA; (c) $d l$-diphenylethylenediamine derivatized with L-FDLA; (d) meso-diphenylethylenediamine derivatized with L-FDLA.
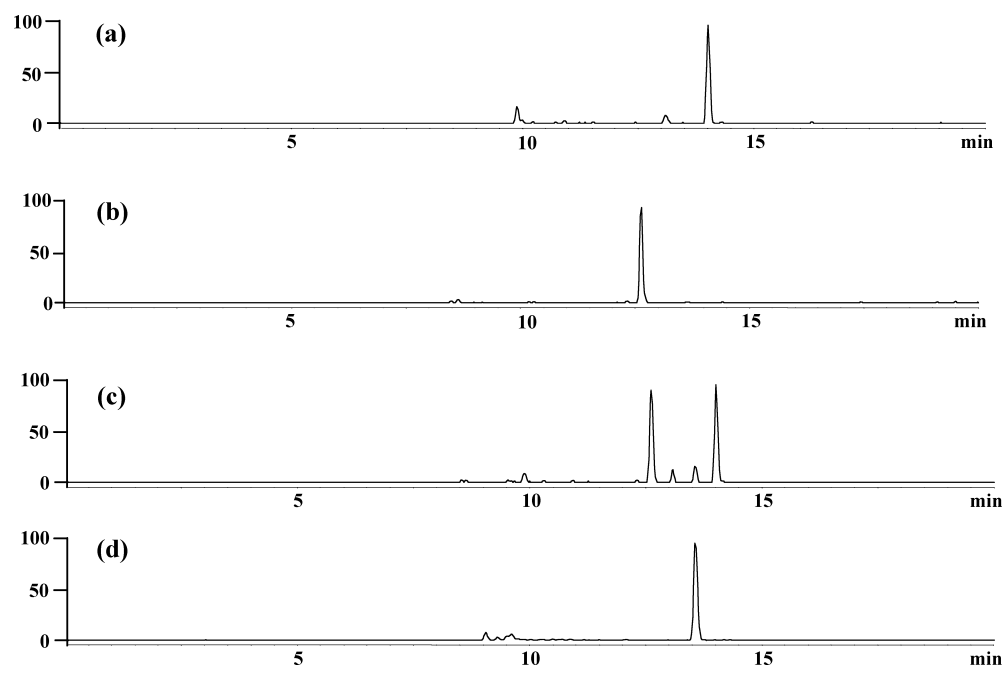

Fig. 3. Mass chromatograms of the $[\mathrm{M}-\mathrm{H}]^{-}$at $\mathrm{m} / z 799$ for (a) $(\mathrm{S}, \mathrm{S})$-diphenylethylenediamine derivatized with D-FDLA; (b) $(R, R)$-diphenylethylenediamine derivatized with D-FDLA; (c) $d l$-diphenylethylenediamine derivatized with D-FDLA; (d) meso-diphenylethylenediamine derivatized with D-FDLA.
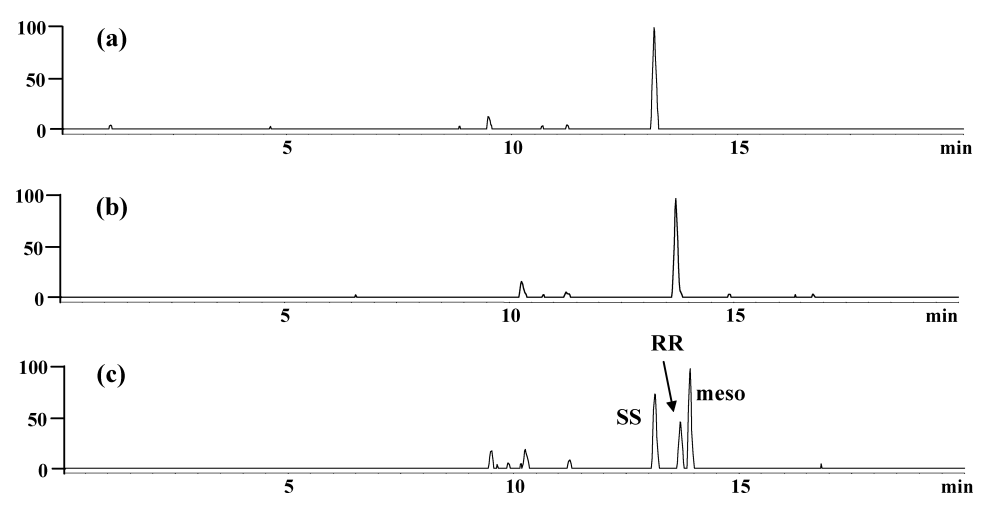

Fig. 4. Mass chromatograms of the $[\mathrm{M}-\mathrm{H}]^{-}$at $\mathrm{m} / z 827$ for (a) (l)-1,2-bis(2-methylphenyl)ethylenediamine derivatized with L-FDLA; (b) (d)-1,2-bis(2-methylphenyl)ethylenediamine derivatized with L-FDLA; (c) (dl)-1,2-bis(2-methylphenyl)ethylenediamine derivatized with L-FDLA.

indicating that it is impossible to determine the absolute configuration by measurement of their optical rotations. Therefore, we prepared three isomers, $d-l-l$, and $d l$-diamine compounds, and determined the absolute configuration of the $l$-isomer to be the $(S, S)$-configuration by an X-ray crystallographic analysis of its 
D- $(+)$-camphorsulfonic acid salt. ${ }^{19)}$

The three $(S, S)-,(R, R)$-, and meso-isomers were subjected to derivatization using FDLA for 1 or $24 \mathrm{~h}$. While the peak heights of the L-DLA derivatives of $d$-, $l$-, and $d l$-isomers after $1 \mathrm{~h}$ were lower than that of the meso-isomer, the three peaks after $24 \mathrm{~h}$ appeared with almost equal peak heights (data not shown). These results suggested a steric hindrance in the 1,2-bis(2methylphenyl)ethylene-1,2-diamine during the FDLA derivatization. The obtained $d$-, $l$-, and $d l$-derivative isomers were derivatized with L-FDLA and the mass chromatograms of the $[\mathrm{M}-\mathrm{H}]^{-}$at $m / z 827$ are shown in Fig. 4 . The $l$-isomer was eluted prior to the $d$-isomer, indicating that the former corresponds to the $(S, S)$ isomer. Figure 4(c) shows the mass chromatograms of the L-DLA derivatives of $d l$-isomer and it was found that this derivative contained the meso-isomer in addition to the $d l$-isomer. The meso-isomer might be formed during the preparation of the optical isomers. As a result of these experiments, the elution order was as follows: $(S, S),(R, R)$, and meso, and the behavior was finally confirmed by the DL-FDLA method. ${ }^{4)}$

3.3 Proposed method for determination of the absolute configuration of 1,2-diaminoethane compounds

The elution behavior of 1,2-diphenylethylene-1,2diamine derivatized with L- and D-FDLA was carefully investigated, and the following pattern was found: 1) $(S, S)$-isomer-L-DLA derivative is eluted prior to the $(R, R)$-isomer-L-DLA derivative; 2$)$ The $(R, R)$ - and mesoisomers-L-DLA derivatives are closely eluted; 3$)$ There is no difference between the retention time of both the L- and D-DLA derivatives of the meso-isomer; 4) This regularity is fundamentally consistent with that of the diamino acids, such as diaminopimelic acid and diaminosuccinic acid except for the elution behavior of the meso-isomer. In this case, because the $(S, S)$-isomer corresponds to the $(\mathrm{L}, \mathrm{L})$-isomer of the diamino acid, the separation behavior can be probably explained using the separation mechanism for advanced Marfey's method. ${ }^{2)}$

Optical resolution is laborious and a suitable methodology is limited. For example, we managed to prepare a single crystal of the complex salt of the $l$-isomer of 1,2 bis(2-methylphenyl)ethylene-1,2-diamine, but not the $d$-isomer, using D-( $d)$-camphorsulfonic acid after several trials of salt formation using tartaric acid, ammonium hexafluorophosphate, or hydrochloric acid. ${ }^{19)}$ For this purpose, advanced Marfey's method is more suitable because it has the following advantages: 1) Operations are easy; 2) Desired peaks can be definitely detected by mass spectrometry; 3) The DL-FDLA method is effective for identification of the isomers; and 4) The absolute configuration can be definitely determined by the elution order.
This method has an additional advantage such that it could be used to monitor the optical resolution process. During optical resolution by repeated recrystallization, the method can always determine the composition of the isomers and monitor the degree of the purified state. Therefore, we believe that this method will contribute to various studies in this field.

\section{References}

1) K. Fujii, Y. Ikai, T. Mayumi, H. Oka, M. Suzuki, and K.-I. Harada, Anal. Chem., 69, 3346 (1997).

2) K. Fujii, Y. Ikai, H. Oka, M. Suzuki, and K.-I. Harada, Anal. Chem., 69, 5146 (1997).

3) Y. Nozawa, N. Sakai, K. Arai, Y. Kawasaki, and K.-I. Harada, J. Microbiol. Methods, 70, 306 (2007).

4) K.-I. Harada, K. Fuji, K. Hayashi, M. Suzuki, Y. Ikai, and H. Oka, Tetrahedron Lett., 37, 3001 (1996).

5) Y. Kawasaki, Y. Nozawa, and K.-I. Harada, J. Chromatogr. A, 1160, 246 (2007).

6) D. Lucet, T. L. Gall, and C. Mioskowski, Angew. Chem. Int. Ed., 37, 2580 (1998).

7) Y. L. Bennani and S. Hanessian, Chem. Rev., 97, 3161 (1997).

8) H. Kim, S. M. So, J. Chin, and B. M. Kim, Aldrichimica Acta, 41, 77 (2008).

9) T. Isobe, K. Fukuda, and T. Ishikawa, Tetrahedron: Asymmetry, 9, 1729 (1998).

10) T. Isobe, K. Fukuda, and T. Ishikawa, J. Org. Chem., 65, 7770 (2000).

11) T. Isobe, K. Fukuda, T. Tokunaga, H. Seki, K. Yamaguchi, and T. Ishikawa, J. Org. Chem., 65, 7774 (2000).

12) T. Isobe, K. Fukuda, K. Yamaguchi, H. Seki, T. Tokunaga, and T. Ishikawa, J. Org. Chem., 65, 7779 (2000).

13) T. Isobe, K. Fukuda, Y. Araki, and T. Ishikawa, Chem. Commun., 243 (2001).

14) T. Ishikawa, Y. Araki, T. Kumamoto, T. Isobe, H. Seki, and K. Fukuda, Chem. Commun., 245 (2001).

15) T. Ishikawa and T. Isobe, Chem. Eur. J., 8, 552 (2002).

16) Y. Kitani, T. Kumamoto, T. Isobe, K. Fukuda, and T. Ishikawa, Adv. Synth. Cat., 347, 1653 (2005).

17) D. Wannaporn and T. Ishikawa, Molecular Diversity, $\mathbf{9}$, 321 (2005).

18) T. Kumamoto, K. Ebine, M. Endo, Y. Araki, Y. Fushimi, I. Miyamoto, T. Ishikawa, T. Isobe, and K. Fukuda, Heterocycles, 66, 347 (2005).

19) A. Ryoda, N. Yajima, T. Haga, T. Kumamoto, W. Nakanishi, M. Kawahata, K. Yamaguchi, and T. Ishikawa, J. Org. Chem., 73, 133 (2008).

20) K. Fujii, T. Shimoya, Y. Ikai, H. Oka, and K.-I. Harada, Tetrahedron Lett., 39, 2579 (1998).

21) X. Sun, S. Wang, S. Sun, J. Zhu, and J. Deng, Synlett, 18, 2776 (2005).

22) H. Kim, C. Yen, P. Preston, and J. Chin, Org. Lett., 8, 5239 (2006).

Keywords: Advanced Marfey's method, FDLA, 1,2-Diaminoethane compounds, Elution order, Optical resolution 